\title{
Chronic hepatitis E after kidney transplantation with an antibody response suggestive of reinfection: a case report
}

\author{
Marcus Panning ${ }^{1 *}$, Kristi Basho $^{1}$, Andreas Fahrner ${ }^{2}$ and Christoph Neumann-Haefelin ${ }^{3}$
}

\begin{abstract}
Background: Hepatitis E virus (HEV) infection is now recognized as a major cause of acute hepatitis worldwide. HEV specific antibodies develop shortly after infection and are thought to confer protection.

Case presentation: We report an immunocompromised patient who developed chronic HEV infection despite the presence of high level antibodies. HEV infection was detected using RT-PCR upon diagnostic evaluation due to increased liver enzymes. Upon retrospective analysis of stored serum samples we found that the patient was HEV RNA positive since 7 months. Chronic HEV infection was successfully treated with ribavirin.

Conclusions: In conclusion, the patient suffered from a chronic course of HEV infection, which was successfully treated with ribavirin. Our case underlines the importance of RT-PCR for HEV diagnostics in immunosuppressed patients and supports the notion that HEV antibodies do not confer universal protection. Counseling patients at risk for chronic HEV infection seems advisable. The role of the humoral and T-cell mediated immune response in cases of HEV reinfection deserves further study.
\end{abstract}

Keywords: Hepatitis E virus (HEV), Antibodies, HEV epitopes, Reinfection, Chronic infection, Ribavirin

\section{Background}

Hepatitis E virus (HEV) infection is increasingly recognized as a major cause of acute hepatitis worldwide. To date, five human pathogenic HEV genotypes are known, of which HEV genotype 3 (gt3) is the dominant HEV genotype in Europe. Recent data demonstrated HEV seroprevalence rates ranging from $<1 \%$ up to $52 \%$ across Europe [1]. HEV gt3 is transmitted zoonotically to humans and infections are linked to the consumption of HEV contaminated meat products. In general, HEV infection remains asymptomatic or presents as mild and self-limiting disease. The humoral immune response begins with the rise of anti-HEV IgM antibodies followed by the development of a robust antiHEV IgG response [2]. The anti-HEV IgG antibody concentration then slowly declines over time but may remain detectable for years $[2,3]$. Although HEV specific antibodies are thought to confer protection against re-infection

\footnotetext{
* Correspondence: marcus.panning@uniklinik-freiburg.de

${ }^{1}$ Institute of Virology, Medical Center - University of Freiburg, Faculty of Medicine, University of Freiburg, Hermann-Herder-Str, 1179104 Freiburg, Germany

Full list of author information is available at the end of the article
}

this topic remains controversial to date. Of note, no definitive minimum protective HEV antibody concentration has been established yet.

In immunosuppressed patients, however, acute HEV gt3 infection can progress to a chronic course (HEV RNA detectable $>6$ months) with high morbidity and mortality rates. Intriguingly, in these patients the HEV specific antibody response is variable or lacking at large.

Here, we report a patient who developed chronic HEV infection shortly after kidney transplantation despite the presence of high anti-HEV IgG pre- and post-transplantation and we describe and characterize the HEV-specific antibody response over time.

\section{Case presentation}

A 64-year-old man with a history of focal segmental glomerulosclerosis underwent kidney transplantation in April 2016. Immunosuppressive medication after transplantation included tacrolimus, mycophenolate mofetil and prednisone. In addition, he received rituximab 750 mg twice in June 2016 and therapeutic plasma exchange (26 times) with albumin and fresh frozen plasma as 
replacement fluid due to recurrence of focal segmental glomerulosclerosis, which subsequently resolved until October 2016. His post-transplantation course showed BKV viremia three months after transplantation (peak viral DNA concentration 383,500 copies/mL). Prednisone was tapered to $10 \mathrm{mg} / \mathrm{d}$ and mycophenolate mofetil was reduced to a dose of $250 \mathrm{mg}$ twice daily. BKV viremia remained below 1000 copies/mL plasma from October 2016 onwards and mycophenolate mofetil was increased to $500 \mathrm{mg}$ twice daily. Intravenous immunoglobulins $(10 \mathrm{~g})$ were given once at the end of June 2016 due to hypogammaglobulinemia. The patient was clinically well and the further course was unremarkable. However, in November 2016, routine laboratory testing revealed elevated AST $(62 \mathrm{U} / \mathrm{L}$, normal range $<50 \mathrm{U} / \mathrm{L})$, ALT $(81 \mathrm{U} / \mathrm{L}$, normal range $<50 \mathrm{U} / \mathrm{L})$, and $\gamma$-GT $(276 \mathrm{U} /$ $\mathrm{L}$, normal range $<60 \mathrm{U} / \mathrm{L}$ ) (Fig. 1). Ultrasound of the liver showed no pathological findings.

In March 2017, he had a routine follow-up visit at the transplant center and his medication included tacrolimus ( $1 \mathrm{mg}$ twice daily, trough level $5-8 \mathrm{ng} / \mathrm{mL}$ ), mycophenolate mofetil (500 $\mathrm{mg}$ twice daily), and prednisone (7.5 mg once daily). He was clinically well and his physical examination was without pathological findings. Still, laboratory tests showed an increased $\gamma$-GT with $215 \mathrm{IU} /$ $\mathrm{L}$, but AST (44 U/L) and ALT $(45 \mathrm{U} / \mathrm{L})$ returned back to normal range. Abdominal ultrasound did not reveal hepatic lesions or signs of liver cirrhosis. Testing for hepatitis $C$ virus RNA was negative, however, HEV RNA was detected using RT-PCR (RealStar HEV RT-PCR Kit 2.0, Altona Diagnostics, Hamburg, Germany) indicating active HEV infection. Concomitantly anti-HEV IgM (71.5 $\mathrm{AU} / \mathrm{mL}$ ) and IgG (149 AU/mL) using ELISA (recomWell IgM/IgG, Mikrogen, Munich, Germany) were positive.
Retrospective analysis of stored plasma samples showed the detection of HEV RNA over the last 7 months beginning in September 2016 consistent with chronic HEV infection (Fig. 1). A fragment of open reading frame (ORF) 1 was amplified according to Johne et al. and phylogenetic analysis of HEV RNA demonstrated an HEV genotype 3c [4]. Interestingly, stored plasma samples taken before kidney transplantation were positive for anti-HEV IgG and negative for anti-HEV IgM as well as HEV RNA indicating resolved HEV infection before transplantation (Fig. 2). Anti-HEV IgG showed a rapid rise after HEV RNA was first detectable in September 2016 whereas anti-HEV IgM remained negative (Fig. 2). It became positive, however, in February 2017. This was accompanied by the detection of anti-HEV IgG antibodies (recomLine HEV IgG, Mikrogen) directed against the Cterminal end of the ORF2 protein (O2CGt1 and O2CGt3) and the full-length ORF3 protein of HEV gt1 and 3 (O3Gt1 and O3Gt3) whereas antibodies against the middle and the N-terminal end of ORF2 were not detectable (Table 1). To better appreciate the level of HEV IgG antibodies we used a quantitiative HEV ELISA (Anti-HEV ELISA, Euroimmun), which was calibrated against the WHO standard and expresses HEV IgG in $\mathrm{IU} / \mathrm{ml}$. Interestingly, we were able to show that the early samples tested HEV IgG borderline (April 2016) and negative (August 2016), respectively (Fig. 2 B). Subsequently, a rise in HEV IgG occurred parallel to the increase seen with the Mikrogen ELISA.

In September 2017, treatment with ribavirin $(200 \mathrm{mg}, 5$ doses daily) was initiated. His liver values were AST (77 U/ L), ALT (78 U/L), and $\gamma$-GT (226 U/L). He received an 11week course of ribavirin (off-label use) and therapy was stopped due to anemia. HEV RNA in plasma was first

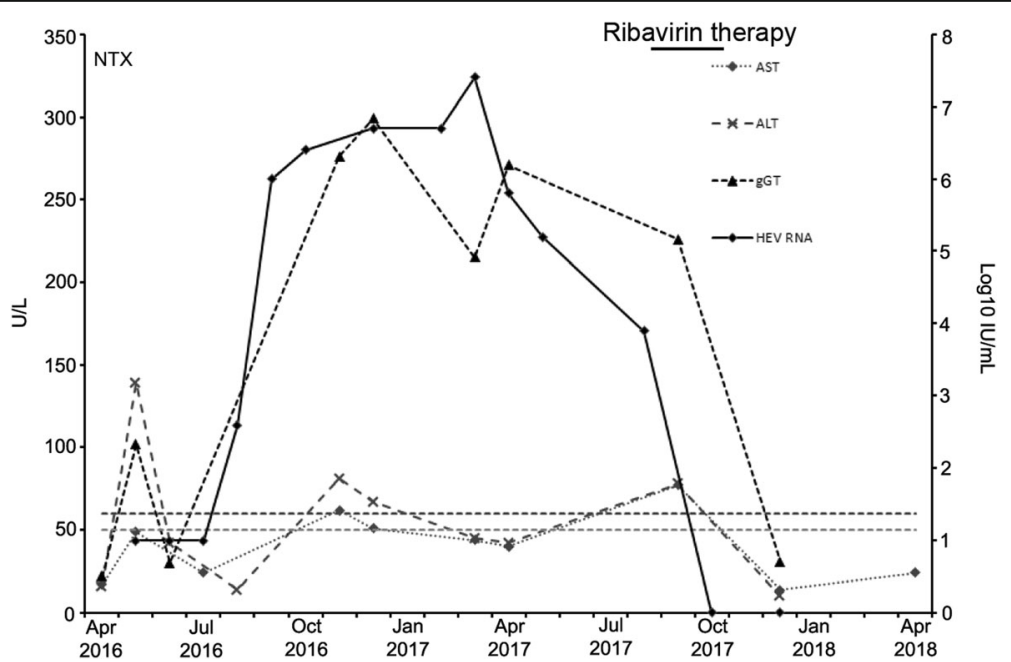

Fig. 1 Time course and level of HEV-RNA concentration and liver enzymes AST, ALT, and YGT after kidney transplantation in April 2016. Vertical broken lines indicate threshold for $\mathrm{y}-\mathrm{GT}$ ( $60 \mathrm{U} / \mathrm{L}$, upper line) and AST, ALT (50 U/L, lower line), respectively 

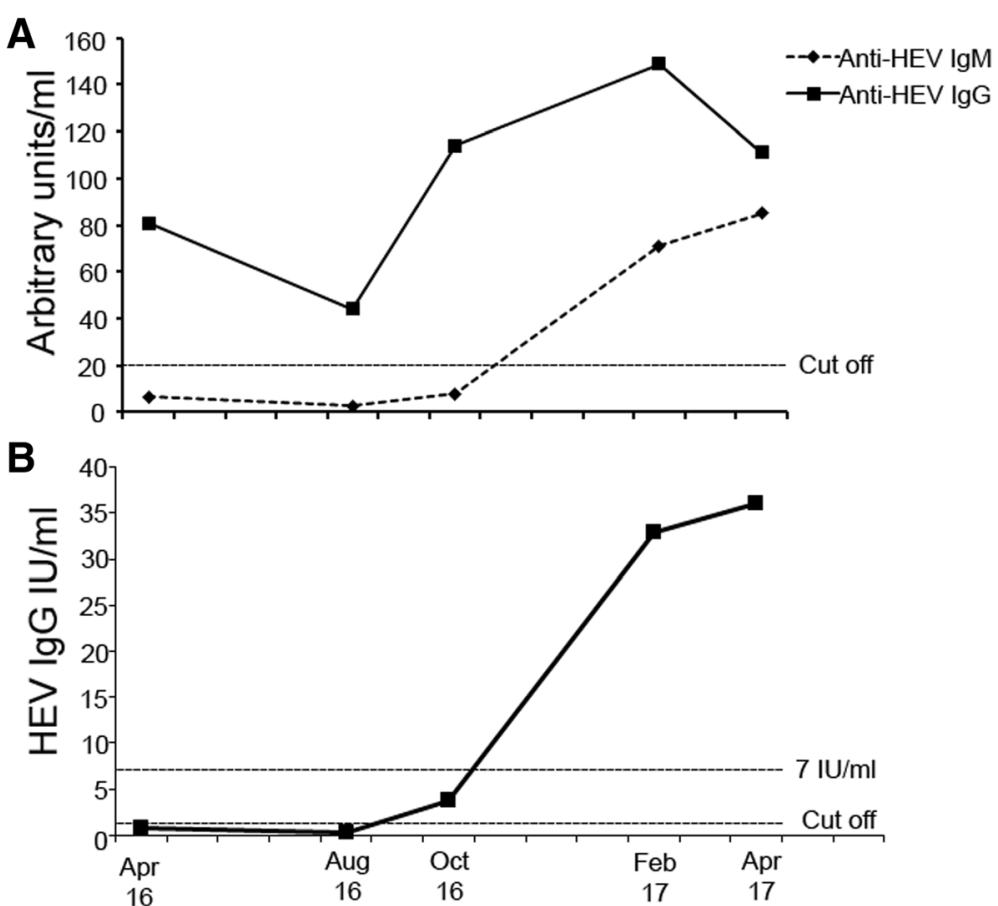

Fig. 2 Development of anti-HEV IgM and IgG over time using HEV-ELISA (Panel a). Development of anti-HEV IgG IU/ml using a quantitative HEV-ELISA (Euroimmun). Solid line denotes HEV-lgG concentration in IU/ml over time. Vertical broken line indicates HEV-lgG concentration of $7 \mathrm{IU} / \mathrm{ml}$. (Panel b)

negative in October 2017 and remained negative indicating successful therapy. Liver enzymes returned back to normal range.

The mode of acquisition of HEV infection in this patient remains unclear but could be related to HEVcontaminated blood products, an HEV-contaminated transplant organ, or food uptake after transplantation. Upon review of the clinical charts we could exclude the administration of blood transfusions shortly before transplantation. The patient had no recent travel. Due to the retrospective nature of the HEV diagnosis no information on food consumption was possible.

\section{Discussion and conclusions}

Here, we were able to show a case of chronic HEV infection despite the presence of detectable anti-HEV IgG before and after kidney transplantation. This suggests HEV reinfection and indicates that $\mathrm{HEV}$ antibodies did not confer protection in this patient.

Cases of HEV reinfection leading to chronic courses have been described anecdotally but comprehensive data is lacking at large [5]. HEV antibodies are considered to be pivotal to control the infection. Recently, HEV antibodies have been standardized and a minimum protective antibody concentration of 2.5 World Health Organization (WHO) units/mL has been proposed [6]. However, a definitive protective titer has not been firmly assessed yet. Choi and coworkers recently demonstrated that rhesus macaques with low HEV-IgG titers $(<6 \mathrm{IU} / \mathrm{ml})$ were not protected against HEV reinfection [7]. Critically, they only analyzed a limited number of animals infected with HEV genotype 1. Of note, in immunocompromised patients a French study found that a low anti-HEV IgG of $<7 \mathrm{WHO}$ units/mL did not protect against reinfection [5]. We used another quantitative assay which was calibrated against the WHO standard

Table 1 Analysis of epitope-specific anti-HEV lgG in consecutive samples

\begin{tabular}{|c|c|c|c|c|c|c|c|}
\hline Month, year & O2NGt1 & O2NGt3 & $\mathrm{O} 2 \mathrm{M}$ & O2CGt1 & $\mathrm{O} 2 \mathrm{CG} 3$ & O3Gt1 & O3Gt3 \\
\hline April 2016 & negative & negative & negative & positive & positive & negative & negative \\
\hline August 2016 & negative & negative & negative & positive & positive & negative & negative \\
\hline October 2016 & negative & negative & negative & positive & positive & positive & positive \\
\hline February 2017 & negative & negative & negative & positive & positive & positive & positive \\
\hline April 2017 & negative & negative & negative & positive & positive & positive & positive \\
\hline
\end{tabular}


and were able to show that anti-HEV IgG was below $7 \mathrm{IU} /$ $\mathrm{ml}$ supporting the hypothesis of non-protective antibody titers in our patient [8]. As a caveat, HEV serological assays differ in their performance and standardization of antibody assays is under debate, e. g. for rubella $[9,10]$. Interestingly, our patient showed a rapid rise in anti-HEV IgG which was followed by an anti-HEV IgM response shortly after. This pattern is typical for a booster immune response and has been demonstrated for HEV in vivo [3]. However, despite the rapid anti-HEV IgG response this did not lead to clearance of HEV RNA from the blood in our patient. This further supports the notion of a limited protective humoral immune response. Interestingly, the rituximab induced B cell depletion might have influenced the development of HEV antibodies in our patient but anti-HEV IgG levels did never fall below the cut-off of the assay. It has been shown that functional B cells are instrumental to control the infection and that antibodies might not be the only correlate of protection [11]. Thus, impaired B cells in our immunosuppressed patient might have contributed to reinfection despite the presence of HEV antibodies.

The epitope-specific analysis of the immune response showed antibodies directed against the C-terminal end of ORF2 which have been regularly detected in another study analyzing the immune response of blood donors [12]. In our patient this was paralleled by an increase of anti-HEV IgG.

Of note, anti-HEV IgG titers decreased shortly after transplantation suggestion an influence of the immunosuppressive therapy on the humoral immune response. Immunosuppressive therapies might also interfere with the HEV replication cycle as recently been demonstrated by Wang and colleagues [13]. They showed that tacrolimus promoted the infection of liver cells with HEV whereas mycophenolate mofetil inhibited HEV replication and steroids showed no effect at all [13]. Our patient received all three drugs giving rise to the notion that the clinical course may have been influenced by his immunosuppressive regimen.

Beyond the humoral response and the immunosuppressive therapy chronic HEV infection has been associated with an impaired HEV-specific T-cell response in transplant patients [14]. However, the T-cell mediated immune response remains largely unclear in HEV infection and deserves further study. We were able to show that the HEV-specific CD8+ Tcell response in chronic HEV patients was diminished in comparison to the acute disease course, but was partially restored after ribavirin therapy (own unpublished data).

Cases of reactivation of HEV have been rarely described to date [15]. We did not detect HEV RNA before and shortly after transplantation arguing against reactivation in our patient.

Clinically important, our patient developed chronic HEV infection which can lead to rapidly developing cirrhosis and liver failure [16]. Treatment with ribavirin was successful in our patient but anemia as well-known side effect of ribavirin led to the discontinuation of antiviral therapy. Currently, ribavirin is the only drug available for treatment of HEV infection and novel treatment strategies are urgently needed. Pegylated interferon-alfa has antiviral activity against HEV, however, cannot be used in patients with stem cell or organ transplants due to a high risk of rejection. In vitro data showed that the drug sofosbuvir, known as a polymerase inhibitor of hepatitis $\mathrm{C}$ virus, may also be effective but beyond case reports no data is available to date [17].

For clinical practice the following points remain important:

I. The presence of anti-HEV IgG may not be protective thus limiting the value of testing for antiHEV IgG before the start of immunosuppressive regimens.

II. Clinical work-up upon increased liver enzymes should include testing for HEV infection irrespective of the anti-HEV IgG status before immunosuppression.

III. In immunosuppressed patients HEV infection should be diagnosed using nucleic acid testing.

IV. Counseling patients at risk for chronic HEV infection regarding the consumption of raw/ undercooked pig products seems advisable.

V. In conclusion, we were able to identify a patient with HEV reinfection who developed a chronic course which was successfully treated with ribavirin. The role of the humoral and T-cell mediated immune response in cases of HEV reinfection deserves further study.

Abbreviations

ALT: Alanine-Aminotransferase; AST: Aspartate-Aminotransferase; BKV: BK virus; gt: genotype; HEV: Hepatitis E virus; IgG: Immunoglobulin G; IgM: Immunoglobulin M; IU/ml: International units/ml; ORF2: Open reading frame 2; RNA: Ribonucleic acid; RT-PCR: Revers-transcription PCR; WHO: World Health Organization; $\gamma$-GT: Gamma-glutyltransferase

Acknowledgements

We are grateful to Ingeborg Hanselmann for excellent technical assistance.

\section{Authors' contributions \\ $\mathrm{AF}$ and $\mathrm{CNH}$ cared for the patient and have been responsible for the clinical part of the manuscript. KB and MP carried out the laboratory investigations and compiled the virological data. MP drafted the initial manuscript, final editing of the manuscript, and coordinated the study. All authors participated in writing and revising the manuscript. All authors have read and approved the final manuscript.}

Funding

This work did not have any external funding.

Availability of data and materials

The datasets used and analyzed during the current study are available from the corresponding author on reasonable request. NCBI GenBank accession number for the HEV ORF1 sequence determined here is MN148916. 
Ethics approval and consent to participate

Not applicable.

\section{Consent for publication}

A written informed consent was obtained from the patient for publication of this case report. All patients' personal data have been anonymized.

\section{Competing interests}

The authors declare that they have no competing interests.

\section{Author details}

'Institute of Virology, Medical Center - University of Freiburg, Faculty of Medicine, University of Freiburg, Hermann-Herder-Str, 1179104 Freiburg, Germany. ${ }^{2}$ Department of Medicine IV, Medical Center - University of Freiburg, Faculty of Medicine, University of Freiburg, Freiburg, Germany. ${ }^{3}$ Department of Medicine II, Medical Center - University of Freiburg, Faculty of Medicine, University of Freiburg, Freiburg, Germany.

Received: 3 May 2019 Accepted: 23 July 2019

Published online: 30 July 2019

\section{References}

1. Hartl J, Otto B, Madden RG, Webb G, Woolson KL, Kriston L, et al. Hepatitis E Seroprevalence in Europe: a meta-analysis. Viruses. 2016;8(8).

2. Kamar N, Dalton HR, Abravanel F, Izopet J. Hepatitis E virus infection. Clin Microbiol Rev. 2014;27(1):116-38.

3. Schemmerer M, Rauh C, Jilg W, Wenzel JJ. Time course of hepatitis Especific antibodies in adults. J Viral Hepat. 2017;24(1):75-9.

4. Johne R, Plenge-Bonig A, Hess M, Ulrich RG, Reetz J, Schielke A. Detection of a novel hepatitis E-like virus in faeces of wild rats using a nested broadspectrum RT-PCR. J Gen Virol 2010;91(Pt 3):750-758.

5. Abravanel F, Lhomme S, Chapuy-Regaud S, Mansuy JM, Muscari F, Sallusto $F$, et al. Hepatitis E virus reinfections in solid-organ-transplant recipients can evolve into chronic infections. J Infect Dis. 2014;209(12):1900-6.

6. Z Zhang J, Zhang XF, Huang SJ, Wu T, Hu YM, Wang ZZ, et al. Long-term efficacy of a hepatitis E vaccine. N Engl J Med. 2015:372(10):914-22.

7. Choi Y, Zhang X, Skinner B. Analysis of IgG anti-HEV antibody protective levels during hepatitis $E$ virus reinfection in experimentally infected rhesus macaques. J Infect Dis. 2019;219(6):916-24

8. Servant-Delmas A, Abravanel F, Lefrere JJ, Lionnet F, Hamon C, lzopet J, et al. New insights into the natural history of hepatitis $E$ virus infection through a longitudinal study of multitransfused immunocompetent patients in France. J Viral Hepat. 2016;23(7):569-75.

9. Vauloup-Fellous C. Standardization of rubella immunoassays. J Clin Virol. 2018;102:34-8

10. Norder H, Karlsson M, Mellgren A, Konar J, Sandberg E, Lasson A, et al. Diagnostic performance of five assays for anti-hepatitis E virus IgG and IgM in a large cohort study. J Clin Microbiol. 2016;54(3):549-55.

11. Kulkarni SP, Sharma M, Tripathy AS. Antibody and memory B cell responses in hepatitis $\mathrm{E}$ recovered individuals, 1-30 years post hepatitis $\mathrm{E}$ virus infection. Sci Rep. 2019;9(1):4090.

12. Kraef C, Schlein C, Hiller J, Westholter D, Denzer U, Horvatits T, et al. Course of HEV viremia and anti-HEV lgM/lgG response in asymptomatic blood donors. J Clin Virol. 2018;105:26-30.

13. Wang Y, Zhou X, Debing Y, Chen K, Van Der Laan LJ, Neyts J, et al. Calcineurin inhibitors stimulate and mycophenolic acid inhibits replication of hepatitis E virus. Gastroenterology. 2014;146(7):1775-83.

14. Suneetha PV, Pischke S, Schlaphoff V, Grabowski J, Fytili P, Gronert A, et al. Hepatitis E virus (HEV)-specific T-cell responses are associated with control of HEV infection. Hepatology. 2012;55(3):695-708.

15. Rivero-Juarez A, Frias M, Lopez-Lopez P, de Los Angeles Risalde M, Brieva T, Machuca I, et al. Hepatitis E virus (HEV) infection in anti-HEV immunoglobulin G-carrying patients after successful hepatitis $C$ virus treatment: reactivation or reinfection? Clin Infect Dis. 2017;64(7):964-6.

16. Kamar N, Selves J, Mansuy JM, Ouezzani L, Peron JM, Guitard J, et al. Hepatitis $\mathrm{E}$ virus and chronic hepatitis in organ-transplant recipients. N Engl J Med. 2008;358(8):811-7.

17. Dao Thi VL, Debing Y, Wu X, Rice CM, Neyts J, Moradpour D, et al. Sofosbuvir inhibits hepatitis $E$ virus replication in vitro and results in an additive effect when combined with ribavirin. Gastroenterology. 2016;150(1):82-5 e4.

\section{Publisher's Note}

Springer Nature remains neutral with regard to jurisdictional claims in published maps and institutional affiliations.
Ready to submit your research? Choose BMC and benefit from:

- fast, convenient online submission

- thorough peer review by experienced researchers in your field

- rapid publication on acceptance

- support for research data, including large and complex data types

- gold Open Access which fosters wider collaboration and increased citations

- maximum visibility for your research: over $100 \mathrm{M}$ website views per year

At BMC, research is always in progress.

Learn more biomedcentral.com/submissions 